\title{
Dietary Supplements and Sports Performance: Metabolites, Constituents, and Extracts
}

\author{
Melvin Williams \\ Department of Exercise Science, Old Dominion University, Norfolk, VA. Address correspondence to \\ mwilliam@odu.edu
}

Received November 17, 2006/Accepted November 23, 2006

\begin{abstract}
This is the fifth in a series of six articles to discuss the major classes of dietary supplements (vitamins; minerals; amino acids; herbs or botanicals; and metabolites/constituents/extracts). The major focus is on efficacy of such dietary supplements to enhance exercise or sports performance. Journal of the International Society of Sports Nutrition. 3(2): 1-5, 2006
\end{abstract}

Key Words: sports supplements, sports nutrition, creatine, HMB.

\section{INTRODUCTION}

Numerous metabolites, constituents, and extracts of plants and animals have been marketed as sports supplements for physically active individuals. In general, these products are theorized to enhance various physiological processes involved in energy production for sport performance. Many of these products consist of single ingredients, while others may be combinations of several substances. This article will focus on two single products, creatine and $\mathrm{HMB}$, which primarily have been marketed as a means to enhance muscle growth, strength and power.

\section{CREATINE}

Creatine is a nitrogen-containing substance found naturally in small amounts in animal foods. In recent years, creatine has been synthesized, mainly as creatine monohydrate, and has been marketed to athletes at all levels. Creatine supplements come in various forms (powder, pills, candy, chews, gels, serum, micronized) for both strength and endurance athletes, including products marketed specifically for males, females, and adolescents. Creatine is a very popular sports supplement. Studies of various athletic groups report usage ranging from 17-75 percent ${ }^{1}$. Relative to the adolescents, in a survey of middle and high school athletes age 10-18 in a New York City suburb found that creatine is being used by athletes at all grades ${ }^{2}$. Use increases with grade level and the prevalence of use by athletes in grades 11 and 12 approaches levels reported among college athletes. The most cited reasons for taking creatine were enhanced performance and appearance. Indeed, creatine continues to be one of the most popular sports supplements of all time.

Oral creatine supplementation, usually as creatine monohydrate, has been reported to increase muscle supplies of free creatine and creatine phosphate (phosphocreatine; PCr), a high-energy phosphagen. A typical creatine loading protocol involves the ingestion of 20 grams of creatine monohydrate over the course of a day, usually in four separate doses of 5 grams each, for 4-6 consecutive days. Casey and Greenhaff ${ }^{3}$ note such a loading protocol will increase muscle total creatine and $\mathrm{PCr}$ levels, but doses beyond 20-30 grams provide no additional benefits. Once loaded, a daily dose of 2-5 grams helps maintain elevated muscle creatine levels. Consuming carbohydrate with creatine appears to increase muscle creatine stores significantly more than creatine supplementation alone ${ }^{4}$. Some individuals, particularly vegetarians with initially low levels of intramuscular creatine, may respond more effectively to creatine supplementation ${ }^{5}$, while individuals with higher intramuscular creatine and phosphocreatine levels and those with fewer type II muscle fibers may be less responsive to supplementation ${ }^{6}$.

Casey and Greenhaff ${ }^{3}$ suggest that the improvements in performance associated with creatine supplementation are due to parallel improvements in ATP resynthesis during exercise as a consequence of 
increased $\mathrm{PCr}$ availability, particularly within the type II muscle fibers. Yquel and others ${ }^{7}$, using magnetic resonance to evaluate $\mathrm{PCr}$ levels during repeated bouts of exercise, indicated that the ergogenic effects also could be attributed to a higher rate of phosphocreatine resynthesis, which would provide more PCr for ATP resynthesis. Creatine is one of the most researched sports supplements, as literally hundreds of studies have evaluated its effects on various types of sport performance. Most of the research has focused on the ability of creatine supplementation to increase muscle mass and related muscular strength and power applicable to performance in very-high-intensity sports, such as sprinting in track events.

Creatine supplementation appears to increase total body and lean body mass; however, short-term gains in total body mass may be primarily water, but longterm gains associated with resistance training appear to be lean muscle mass. Most studies document increases in muscle mass when creatine is supplemented during resistance training ${ }^{8,9}$. The increase in muscle mass may be associated with a creatine supplementation-induced ability to do more repetitions during training, which may induce favorable genetic adaptations in the muscle. For example, Deldicque and others ${ }^{10}$ suggest that the increase in lean body mass often reported after creatine supplementation could be mediated by signaling pathways, such as those involving insulinlike growth factor. In a related vein, Willoughby and Rosene ${ }^{11}$ studied the effect of 12 weeks of creatine supplementation and resistance training on muscular strength and myosin heavy chain (MHC) mRNA and protein expression. Compared to both a control and placebo group, the creatine group significantly increased fat-free mass and strength. Additionally, they found that in general the MHC mRNA and protein expression were significantly higher in the creatine group compared to the other groups, and suggested that the increased strength and muscle size associated with creatine supplementation may be attributed to increase MHC synthesis. Research using muscle biopsy techniques has also shown increases in muscle cell diameter associated with 12 weeks of creatine supplementation and resistance training ${ }^{12}$, which may be associated with the potential for creatine supplementation to amplify the resistance training-induced increase in satellite cell number and myonuclei concentration in skeletal muscle ${ }^{13}$.

Critical reviews of the scientific literature ${ }^{1,14-17}$ including a meta-analysis ${ }^{8}$ and a monograph ${ }^{\prime}$ generally indicate that creatine supplementation may increase muscular strength and endurance as documented by enhanced performance in 1-repetition maximum strength tests, increased number of repetitions in various isotonic and isokinetic resistance exercise tasks, and increased work output during maximal short term (6-30 seconds) cycle ergometer tasks. In general, activities that involve sprinting, jumping or cycling performance show improved performance following creatine supplementation ${ }^{15}$, but the beneficial effects appear to be less consistent ${ }^{18}$. For example, Skare and others ${ }^{19}$, using a standard creatine loading protocol with well-trained male sprinters as subjects, reported significant improvements in 100-meter sprint velocity and time to complete 6 intermittent 60 -meter sprints. Preen and others ${ }^{20}$ reported significant increases in peak power and total work production in 10 sets of multiple 6-second bike sprints with varying periods of recovery in an 80-minute time frame following creatine supplementation. Conversely, Op 'T Eijnde and others ${ }^{21}$ reported no significant improvement in a 70-meter shuttle run sprint power test by welltrained tennis players while Cornish and others ${ }^{22}$ found no beneficial effects on repeated 10-second skating sprints in ice-hockey players.

Some research findings have a direct application to sports competition, such as an increased 1-RM performance in weight lifting and faster 100-meter sprint run times. The laboratory findings for other types of exercise performance are also rather strong, and do support a possible application to actual field competitions. For example, findings of increased muscle power output during intermittent sprint exercises may be applicable to football (soccer) ${ }^{23}$ and other sports associated with high-intensity intermittent sprinting ${ }^{20}$. In one such study, Cox and others ${ }^{24}$ studied the effects of creatine supplementation on an exercise test protocol designed to simulate match play in soccer (football). The test involved 5 blocks of 11-minute exercise involving sprint running, agility runs, and a precision ball-kicking drill interspersed with recovery walks, jogs and runs. Creatine supplementation improved performance in some repeated sprint and agility tasks even though the subjects increased body mass, but the creatine had no effect on ball kicking accuracy. Thus, creatine supplementation might improve speed in repetitive sprints, important for many sports, but may not necessarily enhance sports skills. In support of this latter point, several studies reported no significant effects of creatine loading on tennis skill performance as measured by power and precision of their serves ${ }^{21,25}$. 
Although some forms of creatine have been marketed to endurance athletes, there is limited evidence that creatine supplementation can enhance performance during exercise lasting longer than 90 seconds ${ }^{1,18}$. For example, recent research found that supplementation with Runners Advantage creatine serum, which is marketed to distance runners, had no effect on 5-kilometer run time ${ }^{26}$ and oxidative capacity, substrate utilization, and endurance cycling time trial performance were not enhanced by creatine supplementation ${ }^{27}$.

In summary, Bemben and Lamont ${ }^{15}$ indicate that it generally appears that creatine supplementation does significantly impact force production regardless of sport, sex or age. Kreider ${ }^{16}$ makes the interesting observation that although not all studies report significant results, no studies report ergolytic effects, i.e., that creatine supplementation impairs exercise performance.

\section{HMB (Beta-hydroxy-beta-methylbutyrate)}

HMB is a byproduct of leucine metabolism in the human body. HMB is currently marketed as calciumHMB-monohydrate. Although the ergogenic mechanism is unknown, investigators speculate that HMB may be incorporated into cell components or may influence cellular enzyme activity, in some way inhibiting the breakdown of muscle tissue during strenuous exercise ${ }^{18}$.

Several animal studies involving poultry, cattle, and pigs have indicated HMB supplementation may increase lean muscle mass and decrease body fat. Studies on humans are limited, but the first published report ${ }^{28}$ indicated that HMB supplementation (1.5$3.0 \mathrm{~g} / \mathrm{d}$ ) significantly increased lean tissue and strength in untrained males who initiated a resistancetraining program for three weeks. Research was limited in the following years. In their year 2000 review of research evaluating claims that HMB supplementation can enhance exercise performance, Slater and Jenkins ${ }^{29}$ indicated that at that time there were only two reports published in peer-reviewed journals, and the remaining eight papers were published as abstracts only and had not undergone the peer-review process. Based on these data, they indicated that although there is some support for the claims, at least in young, untrained individuals, the response of resistance-trained individuals is less clear and that there is a need for more tightly controlled, longer duration studies to evaluate the ergogenic effect of HMB supplementation.
Peer-reviewed studies published subsequent to year 2000 are equivocal regarding the ergogenic effect of HMB supplementation on exercise performance in untrained individuals. For example, Panton and others ${ }^{30}$ reported that short-term HMB supplementation (3 grams daily for 4 weeks) during resistance training significantly increased upper body strength in both untrained males and females. Vukovich and others ${ }^{31}$ reported that older subjects engaged in 8 weeks of resistance training and consuming 3 grams HMB daily experienced a significant decrease in body fat, but no significant change in fat-free mass. Jowko and others ${ }^{32}$, in a placebo-controlled study, compared the effects of HMB (3 grams daily) and creatine (20 grams for 10 days; 10 grams for 14 days), both separately and in combination, on body composition and strength of subjects undergoing resistance training for 3 weeks. All subjects gained body mass and strength with training, but the supplement groups gained more than the placebo group. However, the only significant effect on body mass was that of the creatine group compared to the placebo group. Overall these findings with untrained individuals are equivocal, meriting additional research.

In general, studies using resistance-trained individuals as subjects report no significant effects of HMB supplementation. Kreider and others 33 provided forty experienced strength-trained athletes with 0,3 , or 6 grams of HMB daily during four weeks of resistance training, and reported no significant effects of $\mathrm{HMB}$ on muscle tissue breakdown, fat-free body mass, or strength gains in the bench press and leg press. Slater and others ${ }^{34}$ studied the effect of daily HMB supplementation on body composition, muscular strength, and markers of muscle damage and muscle protein turnover following 3 and 6 weeks of training. While both the placebo and HMB groups increased lean body mass and strength with the training, the HMB supplementation provided no additional benefits. Using collegiate football players as subjects, Hoffman and others 35 reported that HMB supplementation ( 3 grams HMB for 10 days) did not increase anaerobic power during 10 days of preseason football training, while Ransone and others ${ }^{36}$, using the same dosage for 4 weeks, reported no significant effect on body composition or muscular strength as measured by bench press, squats, and power cleans.

In a recent review, Palisin and Stacy ${ }^{37}$ indicated that studies of HMB supplementation have shown minimal gains in strength and lean body mass in 
specific populations, mainly untrained athletes. However, HMB use in athletes involved in regular high-intensity exercise has not been proven to be beneficial when multiple variables are evaluated. They concluded that HMB cannot be recommended as an ergogenic until further studies in larger groups reproduce early data.

\section{SUMMARY}

This brief review suggests that the efficacy of various metabolites, constituents and extracts marketed as sports supplements to athletes may vary. Research findings for creatine supplementation are very supportive, while those for HMB supplementation are not. As we shall see in the next and final article of this series, several other dietary supplements may also provide some performance-enhancing effects to certain athletes, but most have not been shown to be effective or have been inadequately studied

\section{REFERENCES}

1. Rawson E, Clarkson, P. Scientifically debatable: Is creatine worth its weight? Sports Science Exchange 16 (4):1-6, 2003.

2. Metzl JD, Small E, Levine SR, Gershel JC. 2001. Creatine use among young athletes. Pediatrics. 108:421-5, 2001.

3. Casey A, Greenhaff, P. Does dietary creatine supplementation play a role in skeletal muscle metabolism and performance. American Journal of Clinical Nutrition 72: 607S-17S, 2000.

4. Snow R, Murphy R. Factors influencing creatine loading into human skeletal muscle. Exercise and Sport Sciences Reviews 31:154-58, 2003.

5. Burke D, Chilibeck PD, Parise G, Candow DG, Mahoney D, Tarnopolsky M. Effect of creatine and weight training on muscle creatine and performance in vegetarians. Medicine \& Science in Sports \& Exercise 36:1946-55, 2003.

6. Syrotuik D, Bell, G. Acute creatine monohydrate supplementation: A descriptive physiological profile of responders vs. nonresponders. Journal of Strength and Conditioning Research 18:610-17, 2004.

7. Yquel RJ, Arsac LM, Thiaudiere E, Canioni P, Manier G. Effect of creatine supplementation on phosphocreatine resynthesis, inorganic phosphate accumulation and pH during intermittent maximal exercise. Journal of Sports Sciences 20:427-37, 2002.

8. Branch JD. Effect of creatine supplementation on body composition and performance: A meta-analysis. International Journal of Sport Nutrition and Exercise Metabolism 13:198-226, 2003.

9. Williams MH, Kreider RB, Branch JD. Creatine: The Power Supplement,. Champaign, IL: Human Kinetics, 1999.

10. Deldicque L, Louis M, Theisen D, Nielens H, Dehoux M, Thissen JP, Rennie MJ, Francaux M. Increased IGF mRNA in human skeletal muscle after creatine supplementation. Medicine \& Science in Sports \& Exercise 37:73136,2005

11. Willoughby D, Rosene J. Effects of oral creatine and resistance training on myosin heavy chain expression. Medicine \& Science in Sports \& Exercise. 33:1674-81, 2001

12. Volek JS, Duncan ND, Mazzetti SA, Staron RS, Putukian M, Gomez AL, Pearson DR, Fink WJ, Kraemer WJ. Performance and muscle fiber adaptations to creatine supplementation and heavy resistance training. Medicine \& Science in Sports \& Exercise 31:1147-56, 1999.

13. Olsen S, Aagaard P, Kadi F, Tufekovic G, Verney J, Olesen JL, Suetta C, Kjaer M. Creatine supplementation augments the increase in satellite cell and myonuclei number in human skeletal muscle induced by strength training. Journal of Physiology 573:525-34, 2006

14. American College of Sports Medicine. The physiological and health effects of oral creatine supplementation. Medicine and Science in Sports and Exercise 32:706-17, 2000.

15. Bemben M, Lamont H. Creatine supplementation and exercise performance: recent findings. Sports Medicine 35:107-25, 2005.

16. Kreider, R. Effects of creatine supplementation on performance and training adaptations. Molecular and Cellular Biochemistry 244:89-94, 2003.

17. Volek J, Rawson E. Scientific basis and practical aspects of creatine supplementation for athletes. Nutrition 20:609-14, 2004.

18. Williams MH. Nutrition for Health, Fitness \& Sports. Boston: McGraw-Hill, 2007.

19. Skare OC, Skadberg BT, Wisnes AR. Creatine supplementation improves sprint performance in male sprinters. Scandinavian Journal of Medicine \& Science in Sports 11:96-102, 2001.

20. Preen D, Dawson B, Goodman C, Lawrence S, Beilby J, Ching S. Effect of creatine loading on long-term sprint exercise performance and metabolism. Medicine \& Science in Sports \& Exercise 33:814-21, 2001.

21. Op 'T Eijnde B, Vergauwen L, Hespel P. Creatine loading does not impact on stroke performance in tennis. International Journal of Sports Medicine 22:76-80, 2001

22. Cornish S, Chilibeck PD, Burke DG. The effect of creatine monohydrate supplementation on sprint skating in ice-hockey players. Journal of Sports Medicine and Physical Fitness 46:90-98, 2006.

23. Hespel P, Maughn RJ, Greenhaff PL. Dietary supplements for football. Journal of Sports Sciences 24:749-61, 2006.

24. Cox G, Mujika I, Tumilty D., Burke L. Acute creatine supplementation and performance during a field test simulating match play in elite soccer players. International Journal of Sport Nutrition and Exercise Metabolism 12:33-46, 2002.

25. Pluim BM, Ferrauti A, Broekhof F, Deutekom M, Gotzmann A, Kuipers H, Weber K. The effects of creatine supplementation on selected factors of tennis specific training. British Journal of Sports Medicine 40:507-12, 2006.

26. Astorino T, Marrocco AC, Gross SM, Johnson DL, Brazil CM, Icenhower ME, Kneessi RJ. Is running performance enhanced with creatine serum ingestion? Journal of Strength and Conditioning Research 19:730-34, 2005. 
27. van Loon LJ, Oosterlaar AM, Hartgens F, Hesselink MK, Snow RJ, Wagenmakers AJ. Effects of creatine loading and prolonged creatine supplementation on body composition, fuel selection, sprint and endurance performance in humans. Clinical Sciences 104:153-62, 2003.

28. Nissen S, Sharp R, Ray M, Rathmacher JA, Rice D, Fuller JC, Connelly AS, Abumrad N. Effect of leucine metabolite $\beta$-hydroxy$\beta$-methylbutyrate on muscle metabolism during resistance-exercise training. Journal of Applied Physiology. 81:2095-2104, 1996.

29. Slater G, Jenkins, D. 2000. Beta-hydroxy-beta-methylbutyrate (HMB) supplementation and the promotion of muscle growth and strength. Sports Medicine. 30:105-16, 2000.

30. Panton LB, Rathmacher JA, Baier S, Nissen S. Nutritional supplementation of the leucine metabolite beta-hydroxy-betamethylbutyrate (HMB) during resistance training. Nutrition. 16:734-9, 2000.

31. Vukovich MD, Stubbs NB, Bohlken RM. Body composition in 70-year-old adults responds to dietary beta-hydroxy-betamethylbutyrate similarly to that of young adults. Journal of Nutrition 131:2049-52, 2001.

32. Jowko E, Ostaszewski P, Jank M, Sacharuk J, Zieniewicz A, Wilczak J, Nissen S. Creatine and beta-hydroxy-beta-methylbutyrate (HMB) additively increase lean body mass and muscle strength during a weight-training program. Nutrition 17:558-66, 2001.

33. Kreider RB, Ferreira M, Wilson M, Almada AL. Effects of calcium beta-hydroxy-beta-methylbutyrate (HMB) supplementation during resistance training on markers of catabolism, body composition, and strength. International Journal of Sports Medicine. 20: 503-509, 1999.

34. Slater G., Jenkins D, Logan P, Lee H, Vukovich M, Rathmacher JA, Hahn AG. Beta-hydroxy-beta-methylbutyrate (HMB) supplementation does not affect changes in strength or body composition during resistive training in trained men. International Journal of Sport Nutrition and Exercise Metabolism. 11:384-96, 2001.

35. Hoffman JR, Cooper J, Wendell M, Im J, Kang J. Effects of beta-hydroxy beta-methylbutyrate on power performance and indices of muscle damage and stress during high-intensity training. Journal of Strength Conditioning Research 18:747-52, 2004.

36. Ransone J, Neighbors K, Lefavi R, Chromiak J. The effect of beta-hydroxy beta-methylbutyrate on muscular strength and body composition in collegiate football players. Journal of Strength and Conditioning Research 17:34-39, 2003.

37. Palisin T, and Stacy, J. Beta-hydroxy-beta-methylbutyrate and its use in athletics. Current Sports Medicine Reports 4:220-23, 2005. 